\title{
A projection approach for multiphase flows
}

\author{
Daniel Hartmann* and Tim Colonius ${ }^{\dagger}$ \\ Division of Engineering and Applied Science \\ California Institute of Technology, Pasadena, CA 91125, USA
}

\begin{abstract}
An Eulerian projection approach for incompressible variable-density two-phase flows is presented. The Navier-Stokes equations governing these flows are reformulated to take the form of the corresponding equations for the lighter phase with a constant density, which can be efficiently solved using standard numerical methods. The effect of the additional mass in the heavier phase is accounted for by a forcing term, which is determined from the solution of an artificial velocity field. This artificial field is subjected solely to inertial and gravity forces as well as the force coupling the flow field and the artificial field. The phase interface in this purely Eulerian approach is described using the level-set method. Results for two-dimensional simulations of the Rayleigh-Taylor instability are presented to validate the new method.
\end{abstract}

\section{Introduction}

Multiphase flows involve moving and deforming embedded interfaces. Their numerical simulation is a great challenge of computational fluid dynamics. Most commonly the flow field is described in an Eulerian frame of reference. In this case, one can broadly distinguish between front capturing and front tracking methods to account for the phase interface. In front capturing methods, the moving phase interface is implicitly represented on a fixed grid that does not conform to the phase boundary. Examples for such approaches are the marker-and-cell (MAC) method, ${ }^{3}$ the volume-of-fluid (VOF) method, ${ }^{4,5}$ the use of shock-capturing schemes, ${ }^{2,6}$ the level-set method, ${ }^{7-9}$ and combined level-set/volume-of-fluid methods. ${ }^{10}$ In front tracking methods, the phase interface is represented explicitly, for example by connected lines in two-dimensional space or by connected triangles in three-dimensional space. ${ }^{11}$

Typically, the aforementioned approaches are used in conjunction with a single set of equations governing the evolution of the flow field in both phases. The fluid in these phases is often assumed to be incompressible ${ }^{8-11}$ and the governing equations are commonly solved using a fractional-step method. ${ }^{12,13}$ In such a projection approach the pressure variable is determined from the divergence-free constraint of the incompressible flow field. Applied to single-phase incompressible flows with constant density and viscosity, this results in a constant-coefficient Poisson equation to be solved, for which efficient numerical methods are available. In the case of a multiphase flow, however, the resulting Poisson equation possesses variable coefficients, making its solution more difficult especially when the material properties significantly vary. ${ }^{11,14}$

For variable-density flows with constant viscosity and no surface tension, Kim and Peskin ${ }^{15}$ present an immersed-boundary method in which the Navier-Stokes equations are formulated for the lightest phase and augmented by a forcing term that accounts for the variable mass in the flow. The forcing term is expressed in terms of a dual set of massless and mass-carrying Lagrangian particles. The approach requires a large number of particles to be distributed in the regions covered by the heavier phases and corresponding interpolation and regularization operators to transfer the velocity field from the Eulerian grid to the Lagrangian points and vice versa.

The objective of the present work is to formulate a purely Eulerian projection approach for a oneequation multiphase model such that the flow equations with constant properties (density and viscosity) can be solved and the effect of the varying properties is accounted for by a forcing term added to the NavierStokes equations. The phase interface is described on the Eulerian grid using the level-set method. However, the method is formulated such that it can be generalized to other techniques for the interface representation.

\footnotetext{
*Postdoctoral scholar, Division of Engineering and Applied Science, California Institute of Technology.

${ }^{\dagger}$ Professor, Division of Engineering and Applied Science, California Institute of Technology.
} 
The structure of this paper is as follows. In section II the set of equations for the new approach is derived from the Navier-Stokes equations for variable-density flows. The numerical method to discretize these equations is summarized in section III, before results are presented in section IV to validate the methodology. Finally, a brief summary is offered in section V.

\section{Governing equations}

The equations governing a two-phase immiscible incompressible flow with variable density $\varrho$ and constant viscosity $\mu$ can be written

$$
\begin{aligned}
\varrho\left(\frac{\partial \mathbf{u}}{\partial t}+\mathbf{u} \cdot \boldsymbol{\nabla} \mathbf{u}\right) & =-\nabla p-\varrho g \mathbf{j}+\mu \nabla^{2} \mathbf{u} \\
\boldsymbol{\nabla} \cdot \mathbf{u} & =0
\end{aligned}
$$

where $\mathbf{u}, p$, and $g$ denote the velocity vector, the pressure, and the gravity constant, respectively. The quantities $\mathbf{x}, t$, and $\mathbf{j}$ denote the position vector, time, and the unit vector in the $y$ direction. The phase interface $\phi_{0}$ is described implicitly using the level-set function $\phi$, which allows us to define three regions in the computational domain $\Omega=\left\{\Omega^{0} \cup \phi_{0} \cup \Omega^{1}\right\}$,

$$
\begin{cases}\phi<0 & \text { for } \mathbf{x} \in \Omega^{0} \\ \phi=0 & \text { for } \mathbf{x} \in \phi_{0} \\ \phi>0 & \text { for } \mathbf{x} \in \Omega^{1}\end{cases}
$$

where $\Omega^{0}$ is the domain covered by the lighter fluid at density $\varrho_{0}$ and $\Omega^{1}$ is the domain covered by the heavier fluid at density $\varrho_{0}+\Delta \varrho$. Using this representation, the spatial density distribution can be expressed as

$$
\varrho(\mathbf{x}, t)=\varrho_{0}+\Delta \varrho H\left(\phi(\mathbf{x}, t)-\phi_{0}\right),
$$

where $H$ is the Heaviside step function defined in terms of the scalar level-set function. The evolution of this function is governed by the level-set equation

$$
\frac{D \phi}{D t}=0,
$$

where $\frac{D}{D t}=\frac{\partial}{\partial t}+\mathbf{u} \cdot \boldsymbol{\nabla}$. Next, we recast the governing equations into a form which allows a solution with efficient standard numerical methods developed for single-phase flows with constant properties, i.e., constant density and viscosity. To this end, let us rewrite Eq. (1a) as

$$
\varrho_{0} \frac{D \mathbf{u}}{D t}=-\nabla p^{\prime}+\mu \nabla^{2} \mathbf{u}-\mathbf{f} H(\phi),
$$

where

$$
\mathbf{f}=\Delta \varrho\left(g \mathbf{j}+\frac{D \mathbf{u}}{D t}\right)
$$

and

$$
\nabla p^{\prime}=\nabla p+\varrho_{0} g \mathbf{j} .
$$

Equation (5) governs the evolution of a fluid with constant properties $\left(\varrho_{0}, \mu\right)$, subject to the effect of the heavier phase represented by the force density f. Let us now decouple Eqs. (5) and (6) and rewrite the full system of equations as

$$
\begin{aligned}
\varrho_{0} \frac{D \mathbf{u}}{D t} & =-\boldsymbol{\nabla} p^{\prime}+\mu \nabla^{2} \mathbf{u}-\mathbf{F} H(\phi), \\
\boldsymbol{\nabla} \cdot \mathbf{u} & =0 \\
\frac{D \tilde{\mathbf{u}}}{D t} & =\frac{\mathbf{F}}{\Delta \varrho}-g \mathbf{j} .
\end{aligned}
$$


The artificial velocity field $\tilde{\mathbf{u}}$ evolves similarly as an inviscid flow subject to the excess gravity force and the force density $\mathbf{F}$, which couples the artificial velocity field $\tilde{\mathbf{u}}$ to the flow field $\mathbf{u}$. The force $\mathbf{F}$ is formulated to drive the flow velocity field $\mathbf{u}$ towards the artificial velocity field $\tilde{\mathbf{u}}$ and vice versa, i.e.,

$$
\mathbf{F}(\mathbf{x}, t)=\varrho_{0} K(\mathbf{u}(\mathbf{x}, t)-\tilde{\mathbf{u}}(\mathbf{x}, t)) .
$$

Eqs. (8) and (9) define a sequence of problems whose solution converges, in the limit of $K \rightarrow \infty$, to the solution of Eqs. (5) and (6). As the parameter $K$, which has the units of an inverse time, is increased, the difference between the velocity and artificial velocity decreases such that the force, $\mathbf{F}$, converges to $\mathbf{f}$, as is demonstrated in section IV. In the limit $K \rightarrow 0$ Eq. (8a) is uncoupled from Eq. (8c), resulting in the equations for the single-phase flow of the lighter phase.

If an explicit time marching scheme is used for the terms involving $\mathbf{F}$, a stiff problem results in the limit of $K \rightarrow \infty$. A von Neumann stability analysis for these terms in the coupled form of Eqs. (8a) and (8c) yields the condition

$$
\Delta t \leq \frac{C}{K} \frac{\Delta \varrho}{\varrho_{0}+\Delta \varrho}
$$

for the time step, where $C$ is a constant that depends on the time marching scheme. This issue will be further discussed in the context of the Rayleigh-Taylor instability test case presented in section IV.A.

\section{Discretization}

\section{III.A. Discretization of the Navier-Stokes equations}

Equations (8a-8c) are discretized by a staggered Cartesian finite-volume method using the implicit CrankNicolson integration for the viscous terms and the explicit second-order Adams-Bashforth scheme for the convective terms as described in Taira and Colonius. ${ }^{17}$ This results in the system of algebraic equations

$$
\left[\begin{array}{ccc}
A & 0 & G \\
0 & \tilde{A} & 0 \\
D & 0 & 0
\end{array}\right]\left(\begin{array}{c}
\mathbf{q}^{n+1} \\
\tilde{\mathbf{q}}^{n+1} \\
p
\end{array}\right)=\left(\begin{array}{c}
\mathbf{r}^{n} \\
\tilde{\mathbf{r}}^{n} \\
0
\end{array}\right)+\left(\begin{array}{c}
\mathbf{b} \mathbf{c}_{1} \\
\tilde{\mathbf{b c}}_{1} \\
b c_{2}
\end{array}\right),
$$

where $\mathbf{q}^{n+1}$ and $\tilde{\mathbf{q}}^{n+1}$ are the discretized flow velocity and artificial velocity flux vectors at time level $(n+1) \Delta t$, respectively, where $\Delta t$ is the time step. Treating the force $\mathbf{F}$ with an explicit Euler scheme, for which $C=1$ in Eq. (10), the right-hand side of Eq. (11) reads

$$
\begin{aligned}
\mathbf{r}^{n} & =\left[\frac{1}{\Delta t} M+\frac{1}{2} L\right] \mathbf{q}^{n}-\frac{3}{2} \mathcal{N}\left(\varrho_{0} \mathbf{q}^{n}\right)+\frac{1}{2} \mathcal{N}\left(\varrho_{0} \mathbf{q}^{n-1}\right)-\mathbf{F}^{n} H\left(\phi^{n}\right), \\
\tilde{\mathbf{r}}^{n} & =\frac{1}{\Delta t} \tilde{M} \tilde{\mathbf{q}}^{n}-\frac{3}{2} \mathcal{N}\left(\Delta \varrho \tilde{\mathbf{q}}^{n}\right)+\frac{1}{2} \mathcal{N}\left(\Delta \varrho \tilde{\mathbf{q}}^{n-1}\right)+\mathbf{F}^{n} .
\end{aligned}
$$

where $M$ is the diagonal mass matrix of the light fluid, $\tilde{M}$ is the mass matrix taking into account the excess mass, $L$ is the discrete Laplacian, and $\mathcal{N}$ denotes the discretized convective term, respectively. The Heaviside function is discretely approximated by the smeared step function ${ }^{8}$

$$
H\left(\phi-\phi_{0}\right) \equiv \begin{cases}1 & \text { if } \phi-\phi_{0}>\alpha \\ 0 & \text { if } \phi-\phi_{0}<-\alpha \\ \frac{\phi-\phi_{0}}{\alpha}+\frac{1}{\pi} \sin \left(\pi\left(\phi-\phi_{0}\right) / \alpha\right) & \text { otherwise, }\end{cases}
$$

where $\alpha=\frac{3}{2} h$ and $h$ is the mesh size. The quantities $\mathbf{b c}_{1}, \tilde{\mathbf{b c}} \mathbf{c}_{1}$, and $b c_{2}$ denote boundary terms and depend on the particular boundary condition which is used. The implicit treatment of the viscous terms is reflected in submatrix $A=\frac{1}{\Delta t} M-\frac{1}{2} L$, whereas for the artificial velocity field $\tilde{A}=\frac{1}{\Delta t} \tilde{M}$. Finally, submatrix $D$ denotes the discrete divergence operator and is constructed such that $G=-D^{T}$. This allows us to make the matrix on the left-hand side of Eq. (11) symmetric and positive definite and to use efficient solution algorithms. The system of equations (11) is solved using a fractional-step algorithm, ${ }^{16}$ which results in the following sub-steps at time level $n \Delta t$ : 
1. Compute the intermediate flow velocity $\mathbf{q}^{\star}$ and the new artificial velocity $\tilde{\mathbf{q}}^{n+1}$

$$
\begin{aligned}
A \mathbf{q}^{\star} & =\mathbf{r}^{n}+\mathbf{b c}_{1} \\
\tilde{A} \tilde{\mathbf{q}}^{n+1} & =\tilde{\mathbf{r}}^{n}+\tilde{\mathbf{b} \mathbf{c}_{1}}
\end{aligned}
$$

2. Solve the Poisson equation to determine the pressure

$$
G^{T} B^{N} G p=G^{T} \mathbf{q}^{\star}-b c_{2},
$$

where $B^{N}$ is the approximate inverse of $A$

$$
A^{-1} \cong B^{N}=\Delta t M^{-1}+\frac{\Delta t^{2}}{2}\left(M^{-1} L\right) M^{-1}+\frac{\Delta t^{3}}{4}\left(M^{-1} L\right)^{2} M^{-1}
$$

3. Correct the intermediate velocities

$$
\mathbf{q}^{n+1}=\mathbf{q}^{\star}-B^{N} G p
$$

Equations (13)-(15) are solved using the conjugate gradient method. Since a Poisson solve representing the computationally most expensive part of the method is only needed for the flow field, the overhead caused by the additional artificial velocity field is comparatively small.

\section{III.B. Discretization of the level-set equation}

The temporal and spatial discretization of the level-set equation follows that described in Hartmann et al. ${ }^{18}$ The level-set Eq. (4) is integrated in time with a 3 -step third-order accurate TVD Runge-Kutta scheme ${ }^{19}$ formulated

$$
\left\{\begin{array}{l}
\phi^{(0)}=\phi^{w}, \\
\phi^{(k)}=\alpha_{k} \phi^{(0)}+\beta_{k} \phi^{(k-1)}-\gamma_{k} \Delta t_{\phi} L\left(\phi^{(k-1)}\right), \\
\phi^{w+1}=\phi^{(N)},
\end{array}\right.
$$

where $k$ is the Runge-Kutta step, $N=3$, and the coefficients $\boldsymbol{\alpha}=\left(0, \frac{3}{4}, \frac{1}{3}\right), \boldsymbol{\beta}=\left(1, \frac{1}{4}, \frac{2}{3}\right)$, and $\boldsymbol{\gamma}=\left(1, \frac{1}{4}, \frac{2}{3}\right)$ are used. The level-set time step $\Delta t_{\phi}$ is equal or a multiple of the flow solver time step and $w \Delta t_{\phi}$ is the time level of the level-set solution. The operator $L(\phi)$ denotes the numerical approximation of the term $\mathbf{u} \cdot \boldsymbol{\nabla} \phi$ in Eq. (4), for which an unlimited fifth-order upstream central scheme ${ }^{20}$ is used. The level-set function is advanced to the next time level when $w \Delta t_{\phi}=n \Delta t$ using the flow velocity field $\mathbf{u}^{n}$ at time level $n \Delta t$. After the level-set solution is obtained at the new time level the flow field is advanced by $i$ time steps until $(n+i) \Delta t=(w+1) \Delta t_{\phi}$. While this technique reduces the strength of the coupling between the level-set solution and the flow solution for $i>1$ it allows us to investigate the performance of the new projection method independently from the properties of the level-set solver. In particular, increasing the value for the parameter $K$ results in a stiffer system (11) and requires a smaller time step be used for the flow solver. However, the level-set solver can be used with the same time step ${ }^{\mathrm{a}}$, such that numerically the representation of the phase interface is the same.

After each level-set time step, the level-set function is reinitialized into a signed distance function by iteratively solving the constrained reinitialization equation ${ }^{21}$

$$
\frac{\partial \phi}{\partial \tau}+S(\tilde{\phi})(|\nabla \phi|-1)=\mathcal{F}
$$

in artificial time $\tau$, where

$$
S(\tilde{\phi})=\frac{\tilde{\phi}}{\sqrt{\tilde{\phi}^{2}+\epsilon^{2}}}
$$

is a smoothed sign function of the level-set function $\tilde{\phi}=\phi(\tau=0)$ before the reinitialization and the smoothing parameter $\epsilon=h$ is set equal to the mesh size. Following Hartmann et al., ${ }^{21}$ Eq. (18) is temporally

${ }^{a}$ This holds true unless the flow field is modified by a variation of $K$ such that the CFL number increases to values above the stability limit of the Runge-Kutta scheme. 
and spatially discretized using a forward Euler scheme and the fifth-order Hamilton-Jacobi WENO scheme of Jiang and Peng. ${ }^{22}$ The forcing term $\mathcal{F}$ on the right-hand side of Eq. (18) corrects the unphysical displacement of the phase interface during the reinitialization. ${ }^{21}$ It is formulated according to the HCR-2 scheme and reads on a uniform grid

$$
\mathcal{F}=\frac{1}{2 h}\left(\tilde{\phi} \frac{\Sigma_{M} \phi}{\Sigma_{M} \tilde{\phi}}-\phi\right)
$$

where $\Sigma_{M}$ is the sum over all neighbor cells across the interface, i.e., cells in which the level-set function possesses the opposite sign. The force is not applied locally where the level-set function on either side of the interface has changed its sign during the reinitialization. Details on the scheme and its derivation are provided in Hartmann et al. ${ }^{18,21}$ Unless stated otherwise, Eq. (18) is solved using 5 iterations after each level-set time step.

\section{Results}

\section{IV.A. Rayleigh-Taylor instability}

To validate the method we present results of a Rayleigh-Taylor instability test case. The configuration is such that a heavier fluid is placed on top of a lighter fluid and the interface between the fluids is initially slightly perturbed. The set-up of our computations matches that of Kim and Peskin, ${ }^{15}$ Fraigneau et al., ${ }^{23}$ and Tryggvason ${ }^{24}$ apart from the fact that Tryggvason reports results for inviscid flow. We consider a $[-0.5 d, 0.5 d] \times[-2 d, 2 d]$ computational domain, where $d$ is the wavelength of an initial corrugation of the light-heavy interface. At the top and the bottom of this domain no-slip boundary conditions are applied, whereas the flow is assumed to be periodic at the left and right boundaries. For the artificial velocity field $\tilde{\mathbf{u}}$ periodic boundary conditions are used at all domain boundaries to avoid imposing additional external forces to this velocity field ${ }^{\mathrm{b}}$. The level-set function is initialized as

$$
\phi(\mathbf{x}, t=0)=-y-0.1 d \cos (2 \pi x / d)
$$

such that the interface location is given by $y(x)=-0.1 d \cos (2 \pi x / d)$. The density distribution in the initially quiescent flow field is prescribed according to Eq. (3) using the smeared Heaviside function (12) and an Atwood number, $A t=\Delta \varrho /\left(2 \varrho_{0}+\Delta \varrho\right)=0.5$. The Reynolds number, $R e=\varrho_{0} \sqrt{d^{3} g} / \mu$, is chosen as $R e=1000$ and length and time are non-dimensionalized by $d$ and $\sqrt{d / g}$, respectively. Unless stated otherwise, a time step $\Delta t=2.5 \times 10^{-4}$ and a parameter value $K=4 \times 10^{3}$ are used. For the level-set solver, a time step $\Delta t_{\phi}=1 \times 10^{-3}$ is used in all computations. In the following, the results are presented with respect to a non-dimensional time $t^{\prime}$ in units of $\sqrt{d /(g A t)}$ following Tryggvason. ${ }^{24}$

Figure 1 illustrates the development of the flow field and the phase interface for a reference solution computed on a $256 \times 1024$ cell grid. This solution can directly be compared with the results of Kim and Peskin, ${ }^{15}$ which were obtained on the same grid using Lagrangian particles to describe density variations in the fluid. The heavier phase can be observed to develop a jet oriented downwards into the lighter fluid. At the front of this jet, the interface begins to roll up at $t^{\prime}=1.25$ forming a mushroom shape embracing a counterrotating vortex pair that is developed until $t^{\prime}=1.75$ matching previously published results. ${ }^{15,23,24}$ In excellent agreement with the solutions of Kim and Peskin ${ }^{15}$ and also Fraigneau et al., ${ }^{23}$ a continuous streak of heavy fluid can be observed to be entrained into the counterrotating vortex pair at time level $t^{\prime}=1.75$. In the ensuing development, the continuing vortex roll-up observed in inviscid simulations ${ }^{24}$ is inhibited by viscous dissipation in agreement with the studies of Kim and Peskin ${ }^{15}$ and Fraigneau et al. ${ }^{23}$ Instead, the vortex pair is stretched in the vertical direction as the jet advances further downwards into the lighter fluid. Along with this vortex pair the rolled-up regions of the interface are stretched and become thinner. At $t^{\prime}=2.0$, parts of the thin end of the mushroom-shaped interface are predicted to be pinched off, while in the solution of Kim and Peskin ${ }^{15}$ (see figure 4 in their paper) the Lagrangian particles form a continuous structure, which is indicative of the heavy fluid being further entrained by the vortex pair. The pinch-off in the present level-set solution occurs where the size of the interface structures becomes of the order of the grid size. Nevertheless, the ensuing generation of a more complex interface structure with small-scale features

b This is similar to the technique used by Peskin ${ }^{25}$ to simulate the flow inside the heart. In these simulations, the heart is embedded in a larger rectangular computational domain with periodic boundary conditions, while the boundary conditions at the heart surface are applied using the immersed-boundary method. 


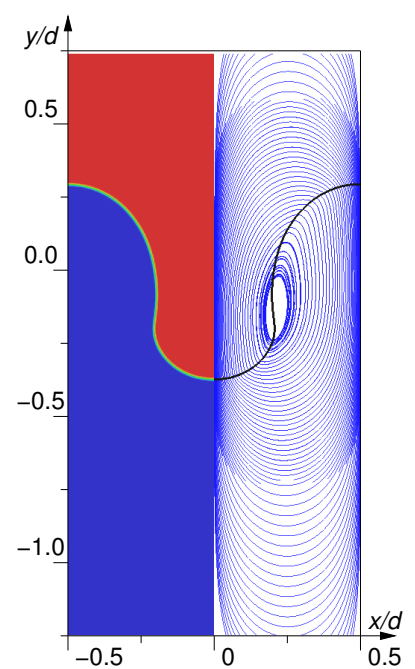

(a)

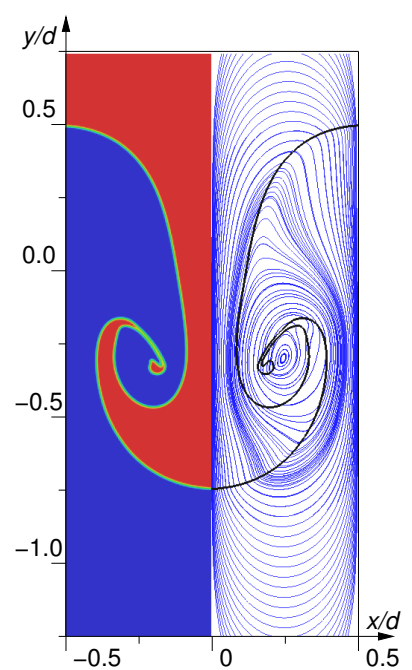

(d)

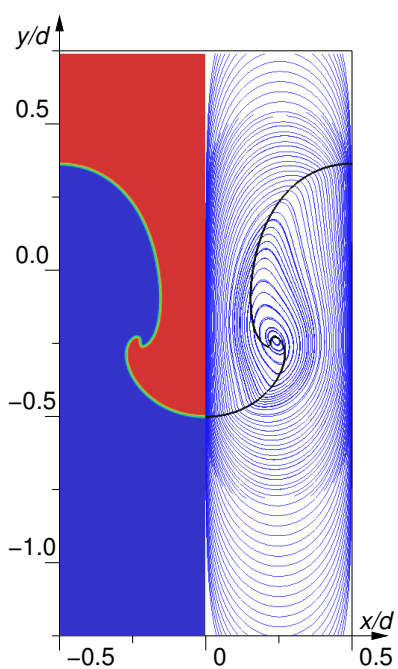

(b)

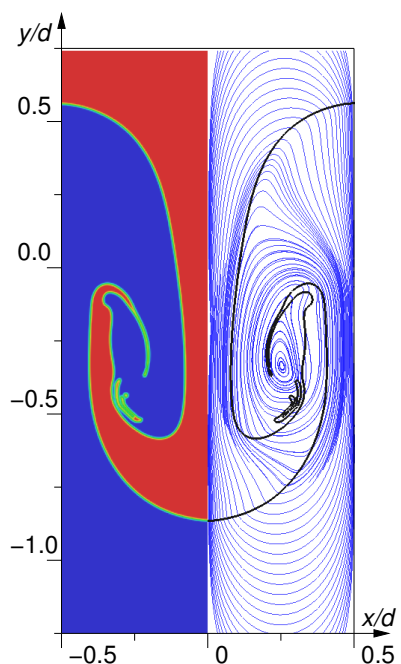

(e)

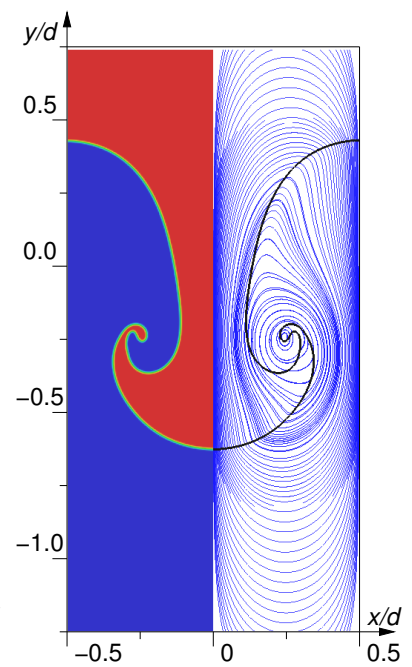

(c)

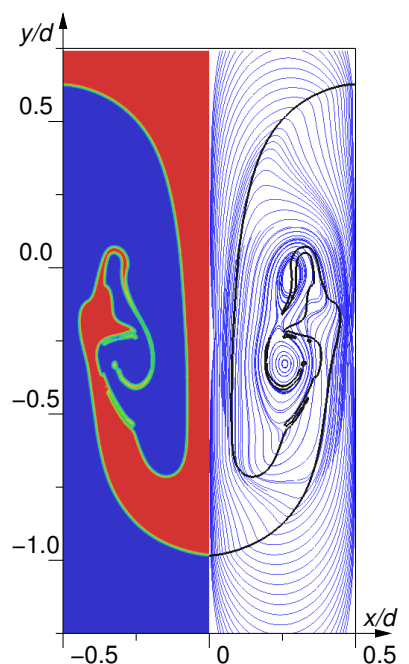

(f)

Figure 1. Rayleigh-Taylor instability at $A t=0.5$ and $R e=1000$ based on the domain width and the gravity constant. Solutions computed on a $256 \times 1024$ cell grid at $(\mathbf{a}) t^{\prime}=1.0 ;(\mathbf{b}) t^{\prime}=1.25 ;(\mathbf{c}) t^{\prime}=1.5 ;(\mathbf{d}) t^{\prime}=1.75 ;(\mathbf{e}) t^{\prime}=2.0 ;(\mathbf{f}) t^{\prime}=2.25$. On the left half of each frame rainbow colors encode the density from $\varrho_{0}$ (blue) to $\varrho_{0}+\Delta \varrho$ (red), while on the right half streamlines and the phase interface $\phi_{0}$ (black contour) are shown.

while the counterrotating vortex pair is further stretched and breaks up into two vortex pairs can be well predicted with the present level-set based method. The solution at time level $t^{\prime}=2.25$ shown in figure 1 (f) agrees convincingly with the results of Kim and Peskin. ${ }^{15}$

Next, we compare simulation results obtained on various grids at time level $t^{\prime}=1.75$, just before small droplets pinch off the thin ends of the mushroom-shaped interface in the reference solution. In figure 2, the interface shapes and flow fields are illustrated for the (a) $32 \times 128$, (b) $64 \times 256$, (c) $128 \times 512$, and (d) $256 \times 1024$ cell grids, the latter of which corresponds to the reference solution. The solutions can be observed to exhibit a more pronounced roll-up and stretching of the interface and to approach the reference solution with increasing grid resolution. Furthermore, an increased grid resolution results in a sharper interface. On the $128 \times 512$ cell grid, the reference solution can be matched reasonably well. This grid is therefore used for all further investigations.

We now turn to show the distribution of the coupling force $\mathbf{F}$ and the impact of different values for the parameter $K$ on these force distributions and the solution for the flow field. The artificial velocity field $\tilde{\mathbf{u}}$ contains the effect of the additional mass in the heavier phase, which is carried over to the flow field through $\mathbf{F}$. Based on the discussion at the end of section II we expect the spatial distribution of $\mathbf{F}$ at a 


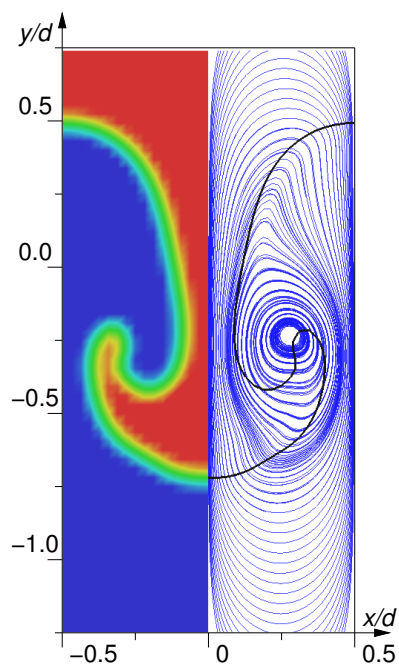

(a)

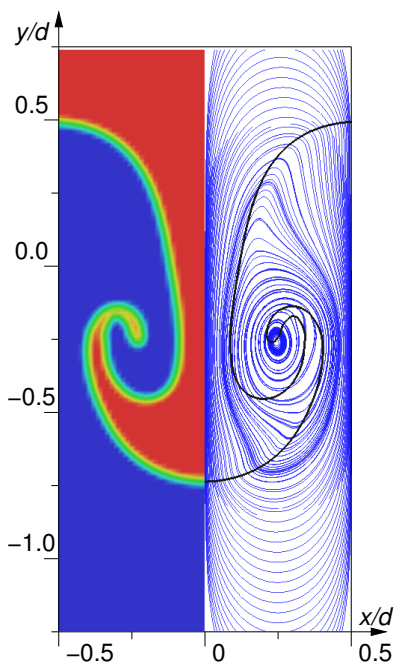

(b)

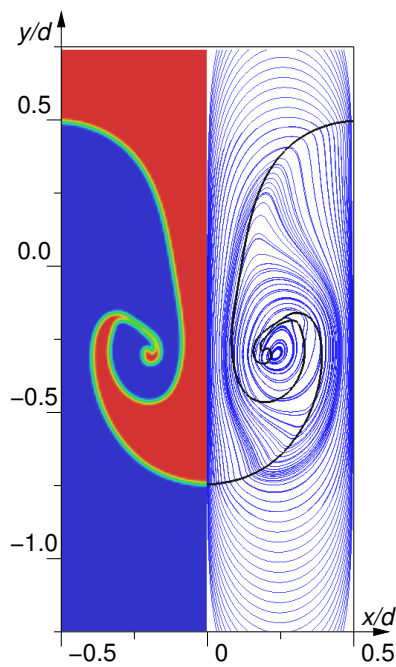

(c)

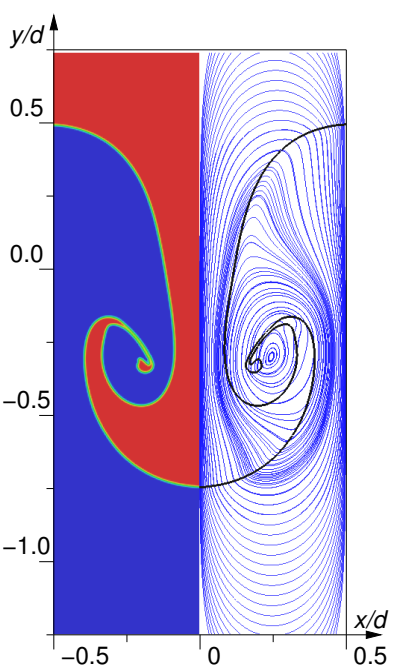

(d)

Figure 2. Solutions at time level $t^{\prime}=1.75$ for the Rayleigh-Taylor instability at $A t=0.5$ and $R e=1000$ on different grids: (a) $32 \times 128$ cell grid; (b) $64 \times 256$ cell grid; (c) $128 \times 512$ cell grid; (d) $256 \times 1024$ cell grid. On the left half of each frame rainbow colors encode the density from $\varrho_{0}$ (blue) to $\varrho_{0}+\Delta \varrho$ (red), while on the right half streamlines and the phase interface $\phi_{0}$ (black contour) are shown.

given time level to converge with increasing $K$. However, owing to the stability condition (10) larger values for $K$ require smaller time steps if an explicit time marching scheme is used. Figure 3 presents the $x$ - and $y$-components of the force $\mathbf{F}$ and the flow field in terms of vorticity contours for various values of $K$ at time level $t^{\prime}=1.75$, for which the phase interface and the flow field are illustrated in figure $1(\mathrm{~d})$. The solutions shown in figure $3(\mathrm{c}),(\mathrm{g})$, and $(\mathrm{k})$ are obtained with the standard parameter $K=4 \times 10^{3}$ that was also used to obtain the reference simulation results presented in figure 1 . The solutions shown in the first and second columns of figure 3 are obtained using smaller parameter values $K=100$ and $K=1 \times 10^{3}$, respectively, while those shown in the fourth column of figure 3 are obtained at a larger parameter value $K=1 \times 10^{4}$.

First, it can be observed that in terms of the interface contour $\phi_{0}$ and the vorticity contours the solutions converge with increasing $K$ (from left to right in figure 3). Second, the spatial distribution of the force $\mathbf{F}$ also converges as $K$ is increased. The solutions and spatial force distributions obtained at $K=4 \times 10^{3}$ and $K=1 \times 10^{4}$ can visually not be discerned in figure $3(\mathrm{c})$ and (d), (g) and (h), and (k) and (l). For smaller values of $K$, differences in the force distributions and the solutions can be observed particularly in the stretched regions of the mushroom-shaped interface, see frames (a), (e), and (i) for the solutions obtained at $K=100$ and (b), (f), and (j) for the solutions obtained at $K=1 \times 10^{3}$. For the smaller values of $K$ the interface exhibits a less pronounced roll-up and is less stretched as compared to the solutions obtained at larger $K$. For the solution obtained at $K=100$, this results in a loss of the finest vortical structures present in the simulations at large $K$, as a comparison of figure 3(i) with $3(\mathrm{k})$ and (l) shows. At these small parameter values the coupling between the mass-carrying artificial velocity field and the flow velocity field is weak and hence, the inertial and gravity forces of the additional mass are insufficiently accounted for particularly in the regions of the heavy phase that exhibit fine topological structures. Recalling that $K$ has units of an inverse time, it is clear that in these regions, where the interface moves and deforms most rapidly and the vorticity attains its maximum absolute values, the solution can only be accurately computed using the larger of the investigated values for $K$. In all the other regions the agreement between the various solutions is excellent, suggesting that the large-scale flow structures can be well resolved using small values for the coupling parameter $K$. As aforementioned, using a larger value for $K$ allows us to take advantage of a larger time step at the cost of inaccurately resolving the small-scale flow structures near the light-heavy interface. The simulations at $K=100$ and $K=1 \times 10^{3}$ were run using a time step $\Delta t=1 \times 10^{-3}$, which corresponds to a CFL number ${ }^{c}$ of 0.22 based on the maximum velocity present in the flow field at time level $t^{\prime}=2.25$. This time step is an order of magnitude larger than the $\Delta t=1 \times 10^{-4}$ used in the simulation with the largest investigated parameter value, $K=1 \times 10^{4}$.

${ }^{\mathrm{c}}$ Note that the CFL number is computed for an instantaneous solution rather than using a characteristic velocity. 


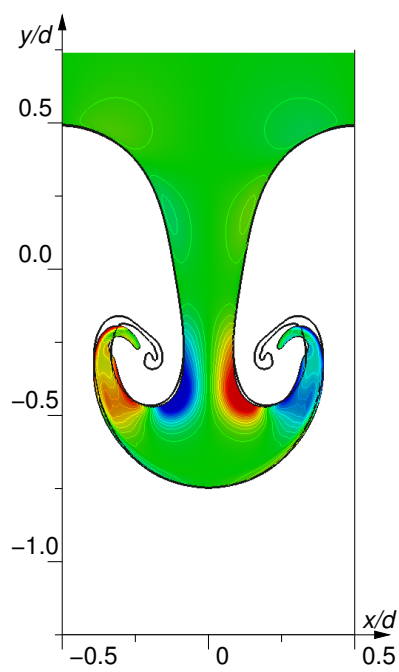

(a)

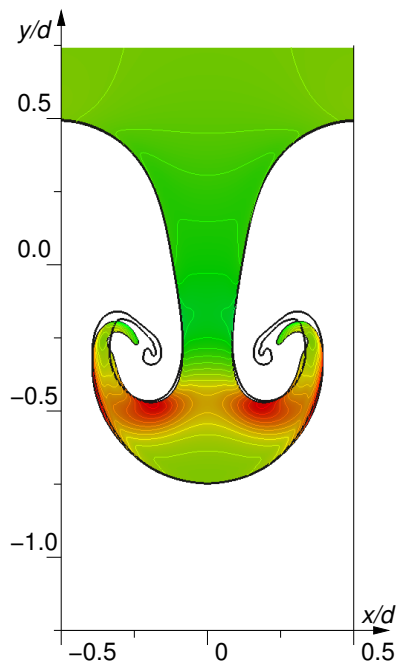

(e)

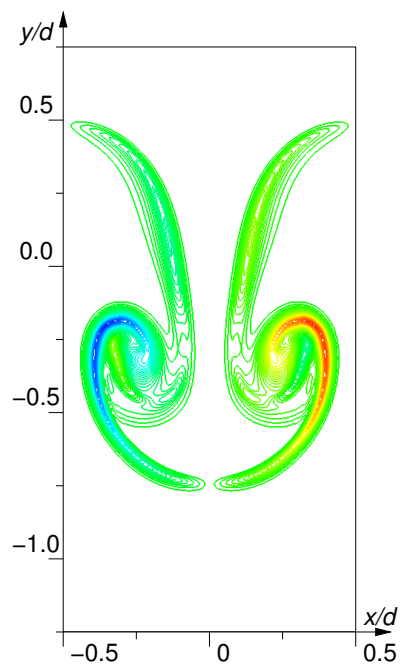

(i)

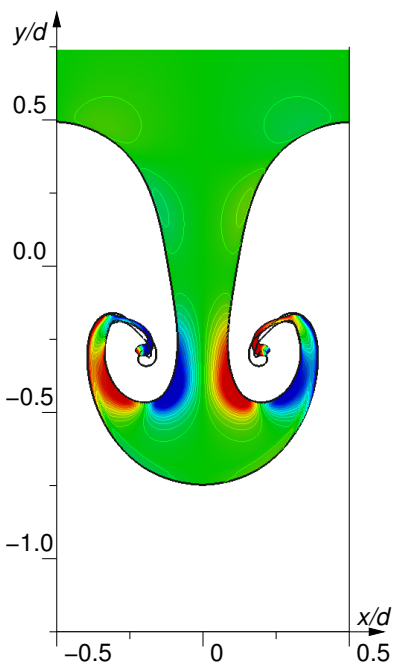

(b)

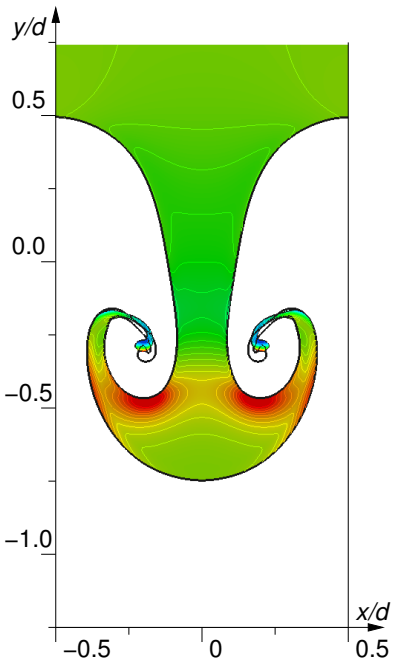

(f)

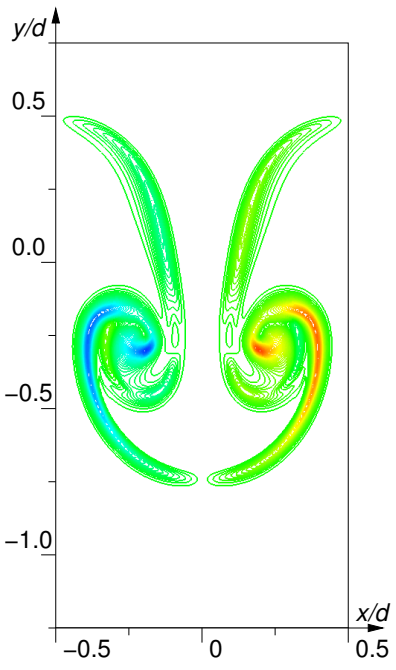

(j)

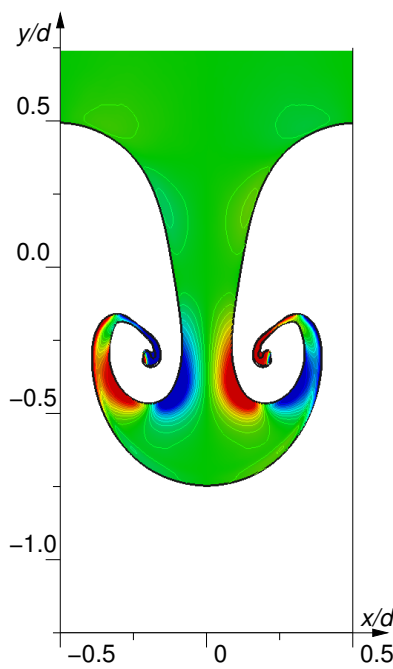

(c)

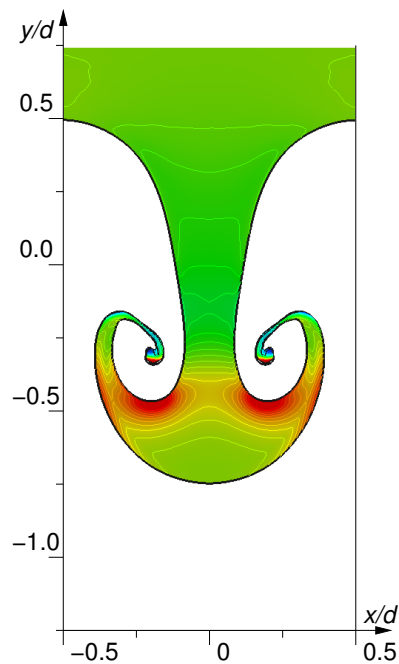

(g)

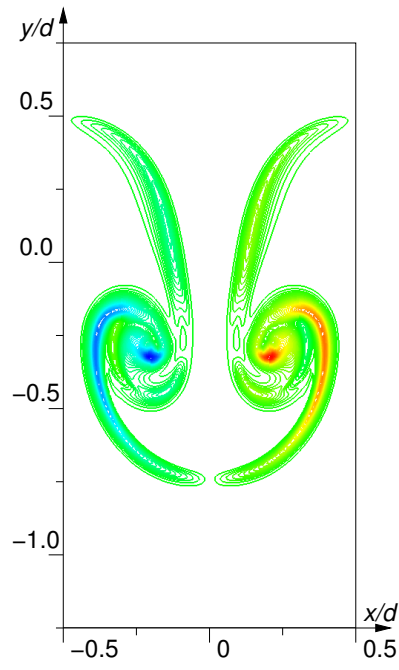

(k)

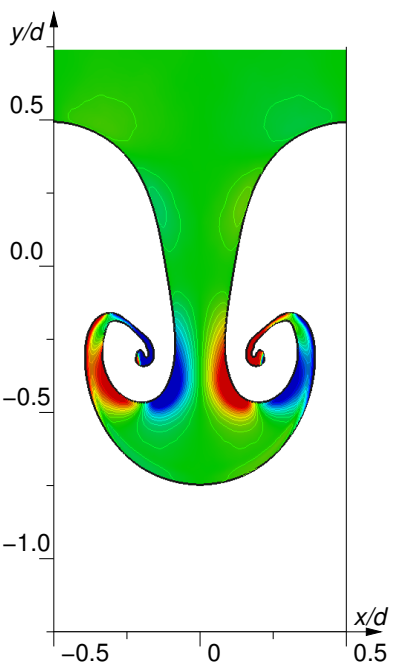

(d)

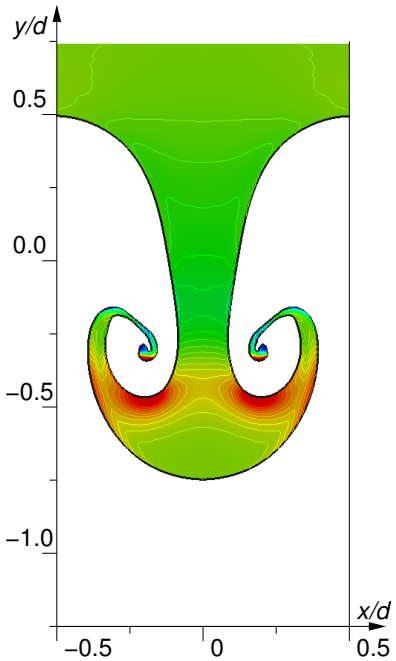

(h)

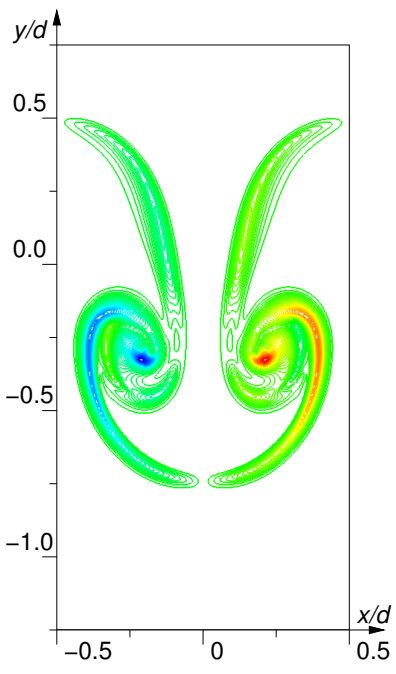

(l)

Figure 3. Parameter study for $K$ on a $128 \times 512$ cell grid (time level $\left.t^{\prime}=1.75\right):(\mathbf{a}, \mathbf{e}, \mathbf{i}) K=100 ;(\mathbf{b}, \mathbf{f}, \mathbf{j}) K=1 \times 10^{3} ;(\mathbf{c}, \mathbf{g}, \mathbf{k})$ $K=4 \times 10^{3} ;(\mathbf{d}, \mathbf{h}, \mathbf{l}) K=1 \times 10^{4}$. The color encodes (a-d) the $x$ - and (e-h) the $y$-components of the force $\mathbf{F}$ in $\Omega^{+}$and (i-l) the vorticity from blue to red in the ranges $-2 \leq F_{x}<0,0<F_{x} \leq 2,-7 \leq F_{y} \leq 7,-40 \leq \omega_{z}<0$, and $0<\omega_{z} \leq 40$, respectively. Contour lines are uniformly spaced at $\Delta F_{x}=0.2, \Delta F_{y}=0.5$, and $\Delta \omega_{z}=1$. Thin black contours (a-h): $\phi_{0}$; thick black contours (a-h): $\phi_{0}$ for $K=1 \times 10^{4}$. 


\section{IV.A.1. Volume conservation}

We now turn to briefly investigate the quality of the representation of the phase interface using the level-set method with an emphasis on volume conservation and the effect of the reinitialization.

The relative volume $V^{1}(t) / V^{1}(t=0)$ of the heavy phase, where

$$
V^{1}(t)=\int_{\Omega} H\left(\phi(\mathbf{x}, t)-\phi_{0}\right) d \mathbf{x},
$$

is plotted over time in figure 4. In figure 4(a), different grid resolutions are investigated. It can be observed that volume is lost on all grids over time. As expected, the volume losses decrease as the grid resolution is increased. Furthermore, it can be observed that volume is well preserved up to time level $t^{\prime}=1.75$, after which the ends of the rolled-up interface become underresolved on the grids. On the $128 \times 512$ cell grid, $0.1 \%$ of the heavy phase volume are lost at this time. Between time levels $t^{\prime}=1.5$ and $t^{\prime}=2.0$ the relative volume can be observed to further decrease when no reinitialization is used, whereas it slightly increases when the reinitialization is used. This is consistent with observations made in an isolated study of the reinitialization process ${ }^{21}$ in which the reinitialization was found to thicken up fine interface structures as they approach the size of the mesh.

In figure 4 (b), the volume loss on the $128 \times 512$ cell grid is compared in simulations at various values of $K$. The aforementioned increase in volume can be observed in all solutions except for that obtained at $K=100$, which is the smallest value investigated for this parameter. As observed in figure 3(a) and (e) for this case, the interface exhibits a less pronounced roll-up and is less stretched in this case as compared to the solutions obtained at larger $K$. Hence, fine interface structures that fall below the limit of grid resolution are not generated to the extent that they can be observed in the simulations with a stronger coupling.

Reinitialization Finally, we briefly investigate the effect of the reinitialization on the development of the flow field and discuss its benefits for level-set based multiphase flow simulations. Solutions with and without reinitialization were computed on a $64 \times 256$ cell grid and the time level $t^{\prime}=2.0$ is considered, at which the ends of the mushroom shape are stretched to such an extent that they cannot be resolved on the computational grid. Figure 5(a) and (c) show the level-set contours obtained without a reinitialization and with constrained reinitialization of the level-set function. Figure 5(a) evidences that the level-set contours are severely compressed indicating steep gradients of the level-set function if no reinitialization is performed. In contrast, we observe in figure 5(c) that if the constrained reinitialization is used, the signed-distance property of the level-set function is well maintained throughout the computational domain. Nevertheless, the characteristic mushroom shape of the phase interface $\phi_{0}$ is quite similar in both solutions and also the entrainment by the counterrotating vortex pair appears to agree well. However, in the present context and in the methods of other authors, ${ }^{8-10,26}$ where the signed-distance property of the level-set function is used to define the (smoothed) density profile in the flow, maintaining this property is essential to obtaining a

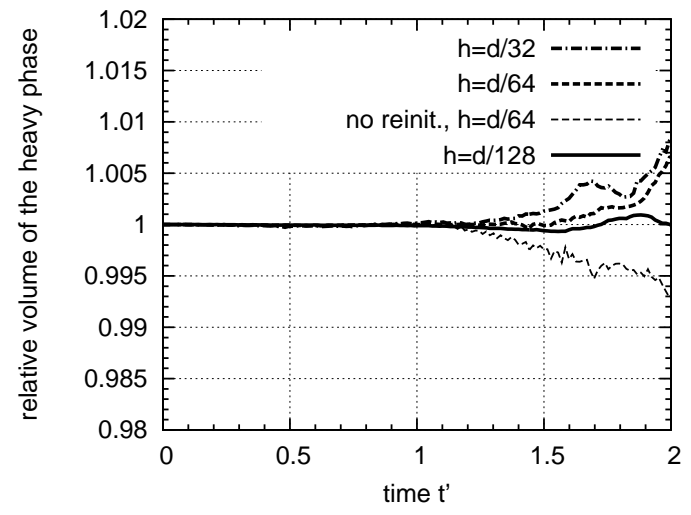

(a)

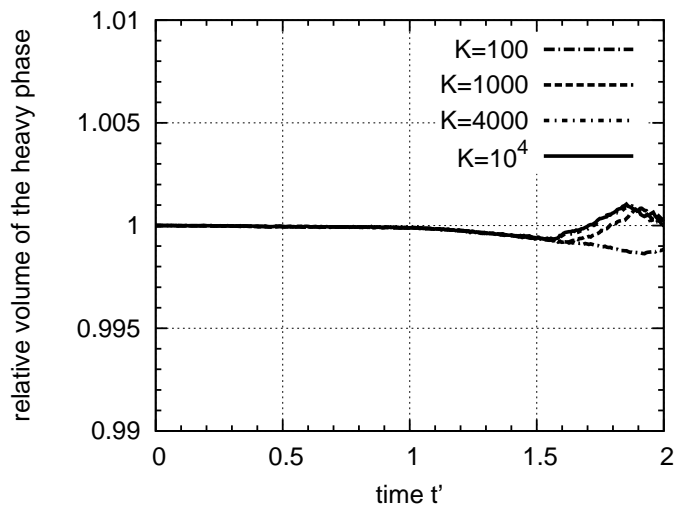

(b)

Figure 4. Relative volume of the heavier phase over time in computations on (a) various grids with different mesh size $h$ and (b) a $128 \times 512$ cell grid using different values for the parameter $K$. 


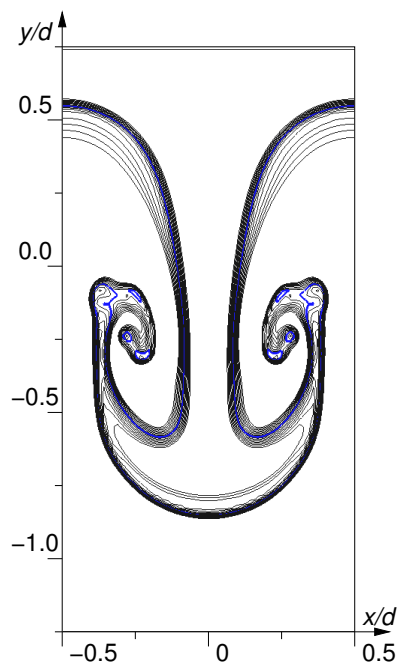

(a)

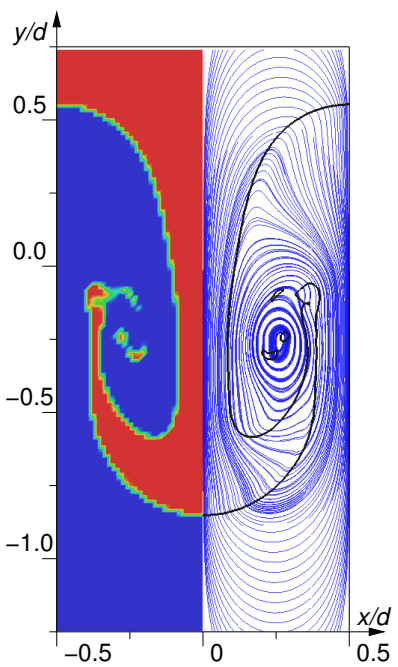

(b)

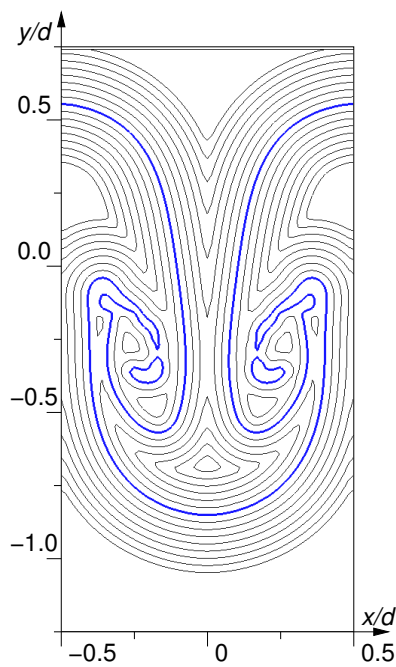

(c)

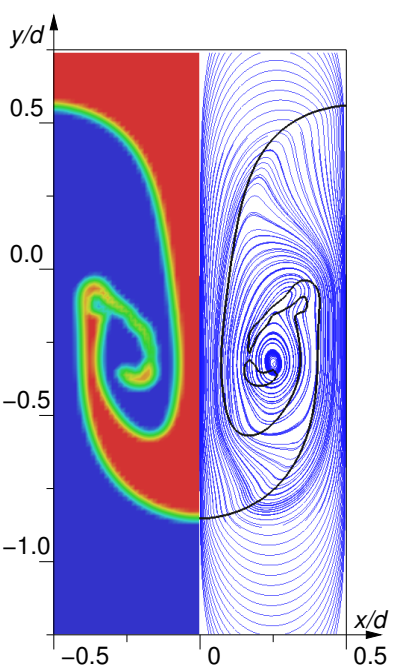

(d)

Figure 5. Comparison of solutions for the Rayleigh-Taylor instability test case computed on a $64 \times 256$ cell grid at $t^{\prime}=2.0(\mathbf{a}, \mathbf{b})$ without reinitialization and $(\mathbf{c}, \mathbf{d})$ with constrained reinitialization of the level set function: (a,c) contours of the level-set function plotted in the range $-0.2 \leq \phi \geq 0.2$ spaced at $\Delta \phi=0.025$; (b,d) left half: density distribution from $\varrho_{0}$ (blue) to $\varrho_{0}+\Delta \varrho$ (red); right half: streamlines and phase interface $\phi_{0}$ (black contour).

uniform and well defined interface thickness ${ }^{\mathrm{d}}$ throughout the flow. An insufficient reinitialization can in these cases have adverse effects on the accuracy of the solution as also discussed in Sussman et al. ${ }^{8}$ This is clearly shown in figure 5(b) in terms of the density distribution obtained without reinitialization. In contrast to the case with constrained reinitialization shown in figure $5(\mathrm{~d})$, the interface has become so thin that the transition from the light fluid to the heavier fluid almost occurs in the form of a jump. Moreover, comparing the density distributions significant differences in the solutions can be observed, which are more difficult to deduce from the level-set contours shown in frames (a) and (c). In the solution obtained with constrained reinitialization, figure 5(d), the counterrotating vortex pair entrains significant amounts of the heavier fluid, resulting in the roll-up of the interface that was also observed in the reference simulation on a finer grid, figure 1. Figure 5(b) shows this entrainment process to be much weaker in the solution obtained without reinitialization, and the roll-up of the interface is clearly less pronounced than the level-set contours suggest.

\section{IV.B. Rayleigh-Taylor instability for a complex perturbed interface}

Finally, we demonstrate the applicability of the proposed method to a more complex problem. A computational domain of size $[-0.5 d, 0.5 d] \times[-1 d, 1 d]$ is discretized by a $256 \times 512$ cell mesh. The flow time step, level-set time step, and the parameter $K$ are prescribed as $\Delta t=2.5 \times 10^{-4}, \Delta t_{\phi}=1 \times 10^{-3}$, and $K=4 \times 10^{3}$, respectively. Following the set-up of Bell and Marcus, ${ }^{27}$ the other parameters are $R e=1000$ and $A t=0.875$, which gives a $7: 1$ density ratio. The level-set function is initialized as

$$
\phi(\mathbf{x}, t=0)=-y-0.00125 d \sum_{j=1}^{8} \cos \left(\lambda_{j} \pi x / d\right),
$$

such that the interface given by $y(x)=-0.00125 d \cos \left(\lambda_{j} \pi x / d\right)$ and $\lambda=(4,14,23,28,33,42,51,59)$ is perturbed by 8 waves of different wave length. The rest of the computational set-up is similar to that described in section IV.A.

The results are presented in figure 6 showing initially an individual growth of several mushroom-shaped interface structures. As they grow, these structures can be observed to interact and merge. These mergers of adjacent structures are naturally handled by the level-set method requiring no additional treatment. At time levels $t=1$ and $t=1.5$ shown in figure 6(b) and (c) several pinched off droplets can be observed to move

\footnotetext{
${ }^{d}$ The thickness of the interface refers to the region around the interface in which the density smoothly varies between the values in the different phases.
} 


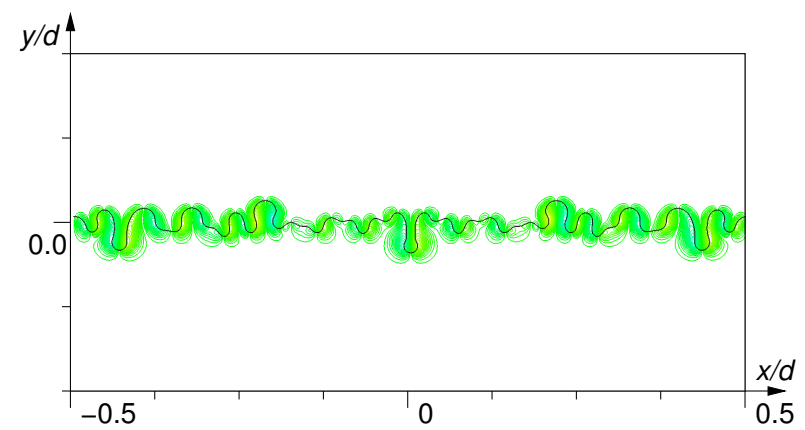

(a)

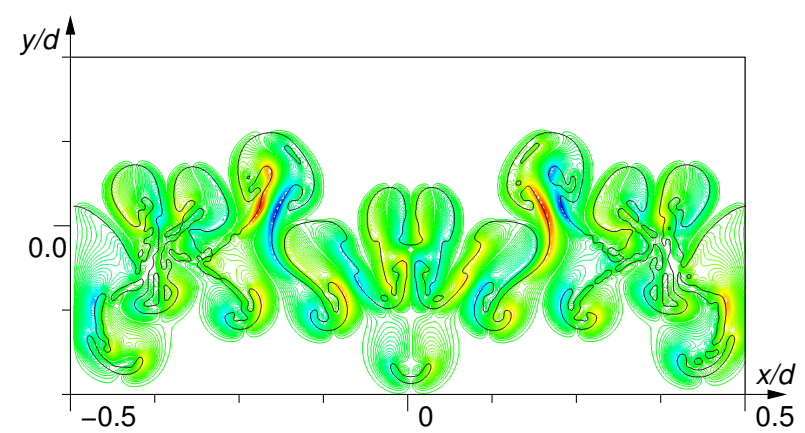

(c)

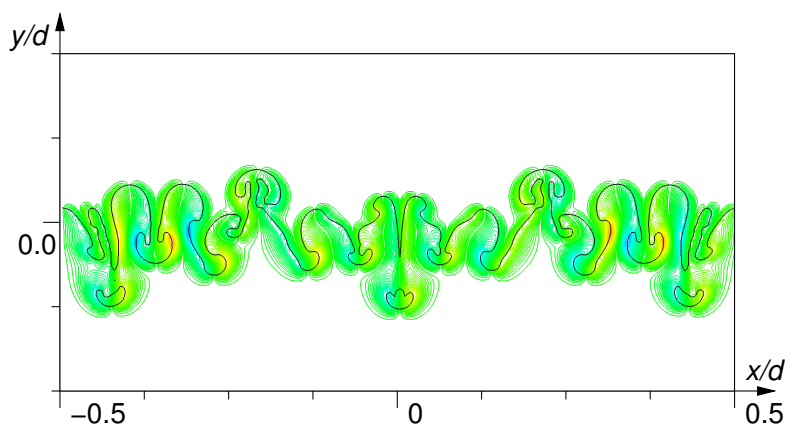

(b)

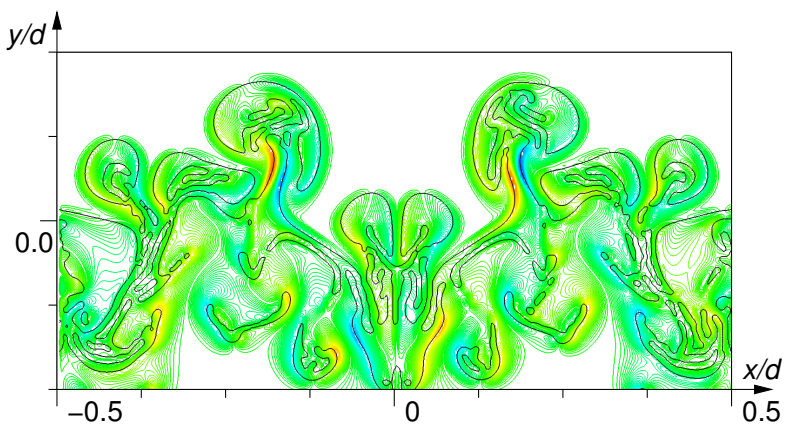

(d)

Figure 6. Complex perturbed density interface: (a) $t=0.5$; (b) $t=1.0$; (c) $t=1.5$; (d) $t=2.0$. Vorticity contours colored from blue to red spaced at $\Delta \omega_{z}=1$ are shown in the range $-50 \leq \omega_{z} \leq 50$. The interface $\phi_{0}$ is shown as the black contour.

away from the main interface accelerated by gravity. At the final time level $t=2$, figure $6(\mathrm{~d})$, the interface is severely distorted and large mushroom-shaped structures have developed pointing into the lighter as well as into heavier fluid.

\section{Summary}

A purely Eulerian projection approach for incompressible variable-density flows was presented. In this approach, the Navier-Stokes equations are solved with the constant properties of the lighter phase, which allows to use efficient standard numerical methods. The effect of the variable density is accounted for by a forcing term, which couples the flow field to an artificial velocity field that carries the additional mass. The forcing term is a function of a free parameter, by which the strength of the coupling can be adjusted. In the limit of an infinitely strong coupling the original Navier-Stokes equations for variable-density flows are recovered. The phase interface is in this purely Eulerian approach described using the level-set method.

The new method was derived and tested for variable-density flows at constant viscosity. Results obtained for a canonical Rayleigh-Taylor instability test case were discussed in detail. In particular, it was shown that the solutions converge when the strength of the coupling between the flow field and the artificial velocity field is increased. These converged solutions agree convincingly with established results from the literature. To demonstrate the capabilities of the formulation for more complex interface shapes results for the evolution of a randomly perturbed density interface were presented. The approach is presently extended for flows with variable density and viscosity in addition to surface-tension effects.

\section{Acknowledgments}

D.H. is sponsored by the German Research Association (Deutsche Forschungsgemeinschaft (DFG)) under grant HA 5535/2-1. The support is gratefully acknowledged. 


\section{References}

${ }^{1}$ Reisman, G., Wang, Y.-C., and Brennen, C., "Observations of shock waves in cloud cavitation," J. Fluid Mech., Vol. 355, 1998, pp. 255-283.

${ }^{2}$ Johnsen, E. and Colonius, T., "Numerical simulations of non-spherical bubble collapse," J. Fluid Mech., Vol. 629, 2009, pp. 231-262.

${ }^{3}$ Harlow, F. and Welch, J., "Numerical calculation of time-dependent viscous incompressible flow of fluid with free surface," Phys. Fluids, Vol. 8, 1965, pp. 2182-2189.

${ }^{4}$ Gueyffier, D., Li, J., Nadim, A., Scardovelli, S., and Zaleski, S., "Volume of fluid interface tracking with smoothed surface stress methods for three-dimensional flows," J. Comput. Phys., Vol. 152, 1999, pp. 423-456.

${ }^{5}$ Popinet, S., "An accurate adaptive solver for surface-tension-driven interfacial flows," J. Comput. Phys., Vol. 228, 2009, pp. $5838-5866$.

${ }^{6}$ Shyue, K., "An efficient shock-capturing algorithm for compressible multicomponent problems," J. Comput. Phys., Vol. 142, 1998, pp. 208-242.

${ }^{7}$ Osher, S. and Sethian, J., "Fronts propagating with curvature-dependent speed: algorithms based on Hamilton-Jacobi formulations," J. Comput. Phys., Vol. 79, 1988, pp. 12-49.

${ }^{8}$ Sussman, M., Smereka, P., and Osher, S., "A level set approach for computing solutions to incompressible two-phase flow," J. Comput. Phys., Vol. 114, 1994, pp. 146-159.

${ }^{9}$ Herrmann, M., "A balanced force refined level set grid method for two-phase flows on unstructured flow solver grids," $J$. Comput. Phys., Vol. 227, 2008, pp. 2674-2706.

${ }^{10}$ Sussman, M. and Puckett, E., "A coupled level set and volume-of-fluid method for computing 3D and axisymmetric incompressible two-phase flows," J. Comput. Phys., Vol. 162, 2000, pp. 301-337.

${ }^{11}$ Tryggvason, G., Bunner, B., Esmaeeli, A., Juric, D., Al-Rawahi, N., Tauber, W., Han, J., Nas, S., and Jan, Y.-J., "A front-tracking method for the computations of multiphase flow," J. Comput. Phys., Vol. 169, 2001, pp. 708-759.

${ }^{12}$ Chorin, A., "Numerical solution of the Navier-Stokes equations," Math. Comput., Vol. 22, 1968, pp. 745-762.

${ }^{13}$ Thémam, R., "Sur l'approximation de la solution des équations de Navier-Stokes par la méthode des pas fractionnaires (I)," Arch. Rat. Mech. Anal., Vol. 32, 1969, pp. 135-153.

${ }^{14}$ Prosperetti, A. and Tryggvason, G., Computational methods for multiphase flow, Cambridge University Press, 2007.

${ }^{15} \mathrm{Kim}, \mathrm{Y}$. and Peskin, C., "Numerical study of incompressible fluid dynamics with nonuniform density by the immersed boundary method," Phys. Fluids, Vol. 20, 2008, pp. 062101.

${ }^{16}$ Perot, J., "An analysis of the fractional step method," J. Comput. Phys., Vol. 108, 1993, pp. 51-58.

${ }^{17}$ Taira, K. and Colonius, T., "The immersed boundary method: A projection approach," J. Comput. Phys., Vol. 225, 2007, pp. 2118-2137.

${ }^{18}$ Hartmann, D., Meinke, M., and Schröder, W., "Differential equation based constrained reinitialization for level set methods," J. Comput. Phys., Vol. 227, 2008, pp. 6821-6845.

${ }^{19} \mathrm{Shu}$, C.-W. and Osher, S., "Efficient implementation of essentially non-oscillatory shock-capturing schemes," J. Comput. Phys., Vol. 77, 1988, pp. 439-471.

${ }^{20}$ Nourgaliev, R. and Theofanous, T., "High-fidelity interface tracking in compressible flows: unlimited anchored adaptive level set," J. Comput. Phys., Vol. 224, 2007, pp. 836-866.

${ }^{21}$ Hartmann, D., Meinke, M., and Schröder, W., "The constrained reinitialization equation for level set methods," J. Comp. Phys., Vol. 229, 2010, pp. 1514-1535.

${ }^{22}$ Jiang, G.-S. and Peng, D., "Weighted ENO schemes for Hamilton-Jacobi equations," SIAM J. Sci. Comput., Vol. 21, 2000, pp. 2126-2143.

${ }^{23}$ Fraigneau, Y., Guermond, J., and Quartapelle, L., "Approximation of variable-density incompressible flows by means of finite elements and finite volumes," Commun. Numer. Methods Eng., Vol. 17, 2001, pp. 893-902.

${ }^{24}$ Tryggvason, G., "Numerical simulations of the Rayleigh-Taylor instability," J. Comput. Phys., Vol. 75, 1988, pp. $253-282$.

${ }^{25}$ Peskin, C., "Numerical analysis of blood flow in the heart," J. Comput. Phys., Vol. 25, 1977, pp. 220-252.

${ }^{26}$ Gómez, P., Hernández, J., and López, J., "On the reinitialization procedure in a narrow-band locally refined level set method for interfacial flows," Int. J. Numer. Meth. Engng, Vol. 63, 2005, pp. 1478-1512.

${ }^{27}$ Bell, J. and Marcus, D., "A second-order projection method for variable-density flows," J. Comput. Phys., Vol. 101, 1992, pp. 334-348. 